\title{
Circular Antenna Array Synthesis with a Differential Invasive Weed Optimization Algorithm
}

\author{
Aniruddha Basak ${ }^{1}$, Siddharth Pal ${ }^{1}$, Swagatam Das ${ }^{1}$ and Ajith Abraham ${ }^{2}$ \\ ${ }^{1}$ Department of Electronics and Telecommunication Engineering,Jadavpur University, Kolkata 700 032, India \\ ${ }^{2}$ Machine Intelligence Resarch Labs (MIR Labs), USA ajith.abraham@ieee.org
}

\begin{abstract}
In this article we describe an optimization-based design method for non-uniform, planar, and circular antenna arrays with the objective of achieving minimum side lobe levels for a specific first null beamwidth and also a minimum size of the circumference. Central to our design is a hybridization of two prominent metaheuristics of current interest namely the Invasive Weed Optimization (IWO) and the Differential Evolution (DE). IWO is a derivative-free real parameter optimization technique that mimics the ecological behavior of colonizing weeds. Owing to its superior performance in comparison with many other existing metaheuristics, recently IWO is being used in several engineering design problems from diverse domains. For the present application, we have modified classical IWO by incorporating the difference vector based mutation schemes from the realm of DE. Three difficult instances of the circular array design problem have been presented to illustrate the effectiveness of the hybrid Differential IWO (DIWO) algorithm. The design results obtained with modified IWO have been shown to comfortably outperform the results obtained with other state-of-the-art metaheuristics like Particle Swarm Optimization (PSO), and Differential Evolution (DE) in a statistically significant fashion.
\end{abstract}

Keywords- antenna arrays, circular arrays, sidelobe suppression, real parameter optimization, metaheuistics, invasive weed optimization, particle swarm optimization, differential evolution.

\section{INTRODUCTION}

In several occasions a single element antenna is unable to meet the gain or highly directive radiation pattern requirements especially suited for long distance communication. Antenna arrays are formed to circumvent such problems by combining many individual antenna elements in certain electrical and geometrical configurations. Antenna arrays have been widely used in diverse applications including radar, sonar, radios, and third generation wireless communication systems $[1-3]$.

The primary design objective of antenna array geometry is to determine the positions of array elements that jointly produce a radiation pattern that matches the desired pattern as closely as possible [4]. In recent past design of non-uniformly spaced linear arrays received a great amount of attention from researchers working in electromagnetic optimization problems. The computational drawbacks of existing numerical methods have forced the researchers all over the world to rely on metaheuristic algorithms founded on simulations of some natural phenomena to solve antenna problems. Since the classical derivative-based optimization techniques are prone to getting trapped in local optima and are strongly sensitive to initialization, many modern metaheuristics were tried to achieve optimized Side Lobe Level (SLL) and null control from the designed arrays, e.g. see [5 10]. However, the design of arrays with other geometrical shapes has not been studied to the same extent, although their importance has been steadily on the rise.

Circular shaped antenna arrays now find various applications in sonar, radar, mobile, and commercial satellite communication systems [11-13]. A first metaheuristic approach towards the design of circular arrays can be traced in the work of Panduro et al. [14] who applied the real-coded Genetic Algorithm (GA) for designing circular arrays with maximal side lobe level reduction coupled with the constraint of a fixed beam width. Shihab et al. in [15] applied the Particle Swarm Optimization (PSO) algorithm that draws inspiration from the intelligent collective behavior of a group of social creatures, to the same problem and achieved better results as compared to those reported in [14]. Recently Panduro et al. [16] compared three powerful population-based optimization algorithms - PSO, GA, and Differential Evolution (DE) on the design problem of scanned circular arrays. The algorithms were compared on a single instantiation of the design problem with number of antenna elements equal to 12 and for a uniform separation of $d=0.5 \lambda$, with an objective of studying the behavior of array factor for the scanning range of $0^{0}$ to $360^{\circ}$ in angular steps of $30^{\circ}$.

In this article we propose to use an improved variant of one recently developed and very powerful metaheuristic algorithm, called the Invasive Weed Optimization (IWO) [17], for designing non-uniform circular arrays with optimized performance with respect to SLL, directivity, and null control in a scanning range of $\left[0^{\circ}, 360^{\circ}\right]$. Since its inception, IWO has found successful applications in many electromagnetic optimization problems like design of E-shaped MIMO Antenna [18], design of compact U-array MIMO antenna design [19], aperiodic thinned aarray antennas [20], time modulated antenna array synthesis [21] etc. In this paper, a modified IWO, whose standard deviation is modulated with individual fitness, has been hybridized with DE [22] and the resulting hybrid algorithm used (for the first time, to the best of our knowledge) to optimize the amplitude excitation and spacing between the elements of a circular antenna array to produce a radiation pattern with optimal performances. Three numerical instantiations of the design problem have been used to illustrate the application of the algorithm. Comparison with the results obtained with other best known real-parameter optimizers like GA, PSO, and DE reflect the superiority of the hybrid Differential IWO (DIWO) in a statistically meaningful way 
where nmi represents the non-linear modulation index. This step ensures that the probability of dropping a seed in a distant area decreases nonlinearly so that the algorithm gradually moves from exploration to exploitation with increasing generations.

Competitive Exclusion: If a plant leaves no offspring then it would go extinct, otherwise they would take over the world. Thus, there is a need of some kind of competition between plants to limit the maximum number of plants in a population. Initially, the plants in a colony will reproduce fast and all the produced weeds will be included in the colony, until the number of plants reaches a maximum value of $p o p \_$max. From then on, only the fittest plants, among the existing ones and the reproduced ones; are taken in the colony and the steps 1 to 4 are repeated until the maximum number of iterations (or function evaluations) have been reached. So, in every generation the population size must be less than or equal to pop_max. This method is known as competitive exclusion and is a selection procedure of IWO.

\section{B. Differential Evolution}

The Differential Evolution (DE), proposed by Storn and Price [24], is also a formidable population-based optimization algorithm. The initial population $W=\left\{W_{1}\right.$, $\left.W_{2}, \ldots, W_{N P}\right\}$ is randomly distributed over the $D$ dimensional search space according to a uniform distribution $w_{\min }^{j} \leq w_{i}^{j} \leq w_{\max }^{j}$ for $j=1,2, \ldots D$ and $i$ $=1,2, \ldots, N P$. After initialization the following steps are executed iteratively:

Mutation: In every generation $G$ a mutation operation is employed to produce a mutant vector $Y_{i}$ with respect to each individual $W_{i}$, the target vector, in the current population $W$. However in literature there are many mutation strategies to create a mutant vector $Y_{i}$ from each target vector $W_{i}$. In DE literature, some frequently used mutation strategies are:

"DE/rand/1": $Y_{i, G}=W_{r_{1}^{i}, G}+F \cdot\left(W_{r_{i}^{i}, G}-W_{r_{3}^{i}, G}\right)$
"DE/best/1": $Y_{i, G}=W_{\text {best }, G}+F \cdot\left(W_{r_{1}^{i}, G}-W_{r_{2}^{i}, G}\right)$

In the above equations the indices $r_{1}^{i}, r_{2}^{i}, r_{3}^{i}, r_{4}^{i}$ and $r_{5}^{i}$ are mutually exclusive integers randomly chosen from [1, $N P]$ and all are different from the base index $i ; F$ is the scaling factor which is a positive number generally chosen in $[0,2]$.

Crossover: For diversity enhancement, a crossover operation is undergone after the generation of donor vector $Y_{i, G}$. The donor vector exchanges its components with the target vector $W_{i, G}$ under this operation to form the trial vector $U_{i, G}=\left[u_{i, G}^{1}, u_{i, G}^{2}, \ldots, u_{i, G}^{D}\right]$. In DE family there are two types of crossover techniques as exponential (or two-point modulo) and binomial (or uniform) [8].

On the other hand binomial crossover is performed as follows:

$$
u_{i, G}^{j}= \begin{cases}y_{i, G}^{j}, & \text { if }\left(\operatorname{rand}_{i, j}[0,1] \leq p_{C r} \text { or } j=j_{\text {rand }}\right) \\ w_{i, G}^{j}, & \text { otherwise }\end{cases}
$$

where $\operatorname{rand}_{i, j}[0,1]$ is a uniformly distributed random number and $p_{C r}$ is the crossover probability.

Selection: This step determines whether the target or the trial vector survives to the next generation $G=G+1$. The selection operation keeps the population size in each generation constant and it can be described as,

$W_{i, G+1}= \begin{cases}U_{i, G}, & \text { if } f\left(U_{i, G}\right) \leq f\left(W_{i, G}\right) \\ W_{i, G}, & \text { if } f\left(U_{i, G}\right)>f\left(W_{i, G}\right)\end{cases}$

where $f(X)$ is the objective function to be minimized.

\section{Modifications on IWO Algorithm}

Modified Standard Deviation Profile: For better performance it is needed that $\sigma_{i t e r}$ should be high in the beginning to have much exploration and after certain generations $\sigma_{i t e r}$ is needed to be small for proper exploitation. To implement this, we have varied the nonlinear modulation index $\left(n \_m \_i\right.$ or $\left.n m i\right)$ with generations as follows:

$$
n_{-} m_{-} i_{\text {iter }}=n m i_{\text {min }}+\left(n m i_{\max }-n m i_{\min }\right) \frac{\text { iter }}{\text { iter }_{\text {max }}},
$$

where $n m i_{\text {min }}$ and $n m i_{\text {max }}$ are typically chosen in the range $(0,15]$. So the equation for standard deviation changes as,

$$
\sigma_{\text {iter }}=\sigma_{\min }+\left(\frac{\text { iter }_{\max }-i \text { ter }}{i t e r_{\max }}\right)^{n_{-} m_{-} i_{\text {iter }}} \cdot\left(\sigma_{\max }-\sigma_{\min }\right) .
$$

The variation of $\sigma_{i t e r}$ with generations is shown in Figure 3. After a number of experiments on the values of $n m i_{\text {min }}$ and $n m i_{\max }$, we have found that the best choice for them is 0.5 and 9 .

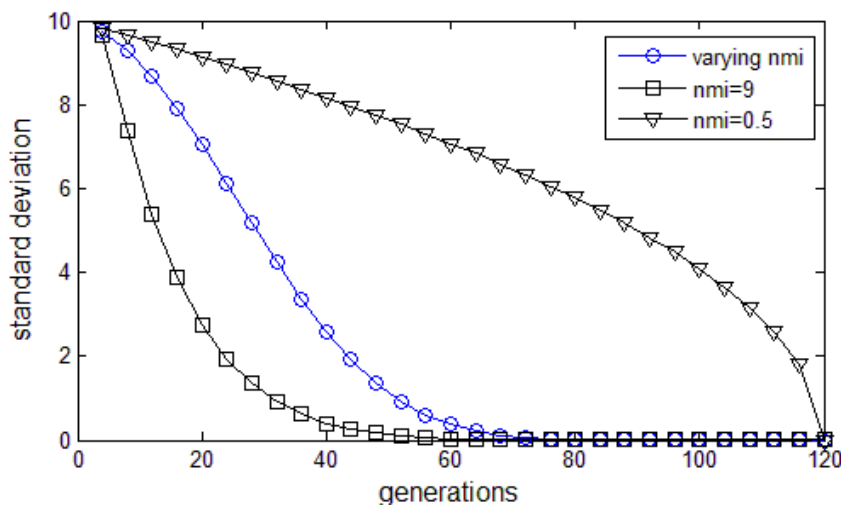

Figure 3: Seed production procedure in the population

Modulation of Standard Deviation with Fitness: We introduced a novel approach of modulating the standard deviation of the weeds with their fitness values. So in one generation the values of $\sigma_{i, i t e r}(i \in[1, N P])$, with which the produced seeds are dispersed, are not the same for all the weeds in the population rather it is made to vary for all the weeds. Considering $\sigma_{i t e r}$ of eqn. (1) as an average value that we assign to the weed $I$ that appears as the median element of the sorted array (sorted according to fitness values) of the weeds at the $t$-th iteration and consider its fitness value to be $F_{\text {median, } t \text {. The weeds having fitness value }}$ less than $F_{\text {median, } t}$ (for minimization) will have $\sigma_{i, \text { iter }}$ less than $\sigma_{i t e r}$ and the others with higher fitness will have higher $\sigma_{i, i t e r}$ compared to $\sigma_{i t e r}$. This is implemented as, 


$$
\sigma_{i, \text { iter }}=\begin{array}{ll}
\left(1+0.5 * \frac{f\left(\vec{X}_{i, \text { iter }}\right)-F_{\text {median, iter }}}{F_{\text {worst,iter }}-F_{\text {median, }, \text { ter }}}\right) \sigma_{\text {iter }} & \text { if } f\left(\vec{X}_{i, \text { iter }}\right) \geq F_{\text {median,iter }}, \\
\left(1-0.5 * \frac{F_{\text {median,iter }}-f\left(\vec{X}_{i, \text { iter }}\right)}{F_{\text {median,iter }}-F_{\text {best, iter }}}\right) \sigma_{\text {iter }} & \text { if } f\left(\vec{X}_{i, \text { iter }}\right)<F_{\text {median,iter }},
\end{array}
$$

where $F_{\text {worstiter }}$ and $F_{\text {bestiter }}$ denote the worst and best fitness in the population respectively at a particular iteration. This provides a chance to the worse weeds in the population to spread its seeds over wide range and find some solution with better fitness.

With these modifications we call this algorithm as MIWO algorithm which is a improved version of the classical one.

\section{Hybridization of M-IWO Algorithm with DE}

To enhance the explorative power of M-IWO algorithm we incorporate the mutation and crossover schemes of the $\mathrm{DE}$ algorithm to produce new seeds from the weeds created by M-IWO.

We have incorporated the features of "DE/best/1/bin" variant with our M-IWO algorithm. Here we describe our whole algorithm:

1. Initialization: A certain number of weeds are randomly spread over the entire search space ( $D$ dimensional). This initial population of each generation will be termed as $X=\left\{x_{1}, x_{2}, \ldots x_{m}\right\}$.

2. Seeds production: Each member of the population $X$ is allowed to produce seeds is allowed to generate seeds according to classical IWO algorithm. Number of seeds to be generated by $\boldsymbol{x}_{\boldsymbol{i}}, i \in[1, m]$ is decided by,

$$
s_{i}=\text { floor }\left(\frac{F_{\max }-f\left(X_{i}\right)}{F_{\max }-F_{\min }} s_{\max }\right),
$$

where $s_{\max }$ is the same as max_seed; and min_seed is taken to be zero. The standard deviation, with which the seeds of each weed will be distributed in the search space, $\sigma_{i, \text { iter }}$ is calculated by equation (10). So for each $\boldsymbol{x}_{\boldsymbol{i}}$ we get $s_{i}$ seeds, generated using standard deviation $\sigma_{i, i t e r}$. These seeds along with their parent weeds create a new intermediate population $W$ of size $n$. Then the mutation and crossover operation is applied on each member $\boldsymbol{w}_{\boldsymbol{i}}$, $i \in[1, n]$ to create another population $Z$ as,

$$
\begin{aligned}
\mathbf{y}_{\mathbf{i}} & =\mathbf{w}_{\text {best }}+F\left(\mathbf{w}_{\mathbf{a}_{2}^{i}}-\mathbf{w}_{\mathbf{a}_{3}^{i}}\right), \\
z^{j}{ }_{i} & =y_{i}^{j} \quad, \text { if } \operatorname{rand}(0,1] \leq p_{C r} \\
& =w_{i}^{j} \quad, \text { if } \operatorname{rand}(0,1]>p_{C r}, j=[1, \ldots, D] .
\end{aligned}
$$

3. Partial Selection using DE: Each element in $Z, z_{i}$, is compared with its corresponding parent $w_{i}$ and the fitter solution is chosen to survive. Using this selection procedure a population of $n$ weeds is created and this is named as $P$. So each weed in $P$ can be described by,

$$
\mathbf{p}_{\mathbf{i}}= \begin{cases}\mathbf{z}_{\mathbf{i}}, & \text { if } f\left(\mathbf{z}_{\mathbf{i}}\right) \leq f\left(\mathbf{w}_{\mathbf{i}}\right) \\ \mathbf{w}_{\mathbf{i}}, & \text { if } f\left(\mathbf{z}_{\mathbf{i}}\right)>f\left(\mathbf{w}_{\mathbf{i}}\right)\end{cases}
$$

4. Final Selection of weeds: The members of $P$ are arranged according to increasing fitness (from best to worst) and the only the first $m$ weeds are selected to pass to the next generation.

Steps 2, 3 and 4 are repeated until the maximum function evaluations are reached. Here onwards we will consider the proposed hybrid algorithm as Differential Invasive Weed Optimization (DIWO).

\section{Simulation Results}

In this paper we will consider three problem instances. We have tried to find the optimal array pattern using a hybrid invasive weed differential evolution algorithm. The results are compared with invasive weed algorithm, particle swarm and differential evolution. A comparison of the results obtained by various algorithms is needed to justify the need of using a hybrid algorithm.

The parameters chosen for the DIWO algorithm are as follows: $n m i_{\min }, n m i_{\max }=0.5,9 ; \sigma_{\min }, \sigma_{\max }=0.001,5$; max_seed, min_seed $=6,0 ; F, p_{C r}=0.5,0.8 ;$ iter $_{\max }=500$; $m$ (Initial population size $)=100 ;$ pop_max $=200$.

For IWO we used the following parametric setup for all the design examples considered here: $s d_{\max }=0.1, s d_{\min }=10^{-5}$, initial number of plants $=100$, maximum number of seeds $=5$ and maximum plants $=$ 200. For the competitor algorithms PSO and DE we used the best possible parametric setup as explained in the relevant literatures.

\section{Case A. 12 element array}

In this problem instance we are required to find the optimal pattern for 12 element array with desired maximum at $180^{\circ}$ and no null control.

Table 1: Results for median of 25 trials (Case A)

\begin{tabular}{|c|c|c|}
\hline Algorithm & SLL $(\mathrm{dB})$ & Directivity $(\mathrm{dB})$ \\
\hline DIWO & $\mathbf{- 2 2 . 2 8}$ & $\mathbf{1 1 . 6 2}$ \\
\hline IWO & -19.43 & 11.52 \\
\hline PSO & -18.64 & 11.31 \\
\hline DE & -18.61 & 11.29 \\
\hline
\end{tabular}

Table 2: Objective function and $P$-values for Wilcoxon's rank-sum test (Case A)

\begin{tabular}{|c|c|c|c|}
\hline Algorithm & Mean obj. value & Std. Dev & $P$-values \\
\hline DIWO & $\mathbf{0 . 1 7 1 4}$ & $\mathbf{0 . 0 2 2 1}$ & NA \\
\hline IWO & 0.2013 & 0.0349 & $4.323 \mathrm{e}-08$ \\
\hline PSO & 0.2241 & 0.0432 & $8.113 \mathrm{e}-13$ \\
\hline DE & 0.2176 & 0.0311 & $3.566 \mathrm{e}-15$ \\
\hline
\end{tabular}

The array pattern obtained is shown in Figure 4. From the results it is clear that DIWO finds an array pattern with the best minimized sidelobes and greatest directivity. From Table 2 we conclude that the results obtained from DIWO are statistically significant according to the Wilcoxon's ranksum test $[23,24]$. 


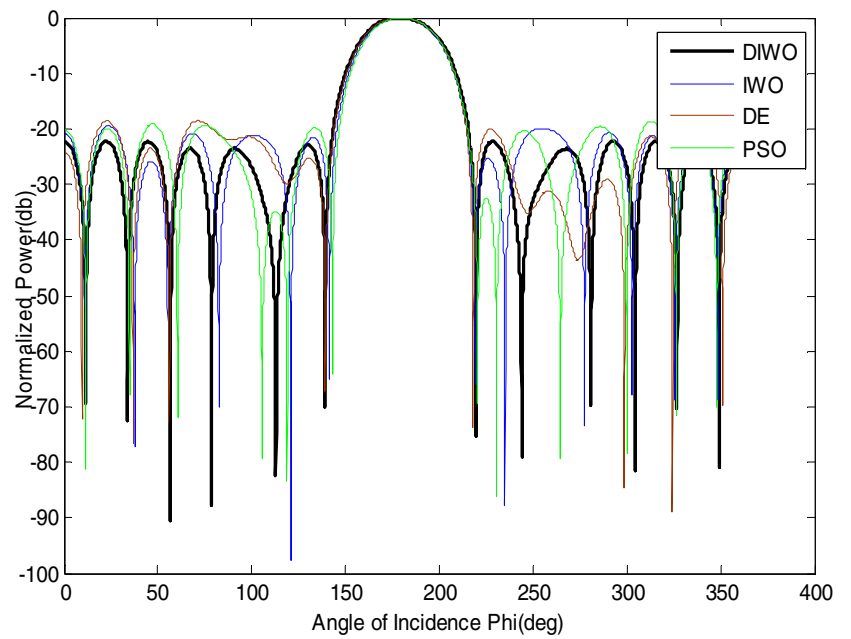

Figure 4: Array Pattern Plot (Case A)

Case B.12 element array with null at $50^{\circ}$

In this problem instance we are required to find the optimal pattern for 12 element array with desired maximum at $180^{\circ}$ and null at $50^{\circ}$.

Table 3: Results for median of 25 trials (Case B)

\begin{tabular}{|c|c|c|c|}
\hline Algorithm & SLL(dB) & Directivity $(\mathrm{dB})$ & $\mathrm{AF}$ at $50^{\circ}(\mathrm{db})$ \\
\hline DIWO & $\mathbf{- 2 1 . 1 6}$ & $\mathbf{1 1 . 3 4}$ & $\mathbf{- 7 7 . 0 4 d B}$ \\
\hline IWO & -18.32 & 11.21 & $-51.42 \mathrm{~dB}$ \\
\hline PSO & -17.83 & 10.87 & $-69.43 \mathrm{~dB}$ \\
\hline DE & -18.13 & 11.08 & $-47.44 \mathrm{~dB}$ \\
\hline
\end{tabular}

Table 4: Objective function and $P$-values for Wilcoxon's rank-sum test (Case B)

\begin{tabular}{|c|c|c|c|}
\hline Algorithm & Mean obj. value & Std. Dev & $P$-values \\
\hline DIWO & $\mathbf{0 . 1 7 6 7}$ & $\mathbf{0 . 0 2 8 9}$ & NA \\
\hline IWO & 0.2167 & 0.0331 & $4.923 \mathrm{e}-11$ \\
\hline PSO & 0.2243 & 0.0487 & $8.954 \mathrm{e}-11$ \\
\hline DE & 0.2178 & 0.0318 & $4.283 \mathrm{e}-11$ \\
\hline
\end{tabular}

From the results it is clear that DIWO finds an array pattern with minimum sidelobes and greatest directivity. DIWO also suppresses the array pattern to $-77.04 \mathrm{~dB}$ at the required null direction of $50^{\circ}$. From Table 4 we conclude that the results obtained from DIWO are statistically significant.

Case C. 12 element array with nulls at $50^{\circ}$ and $120^{\circ}$

In this problem instance we try to find the optimal array pattern with minimum sidelobes, maximum directivity, maximum radiation power at $180^{\circ}$ and array pattern nulls at $50^{\circ}$ and $80^{\circ}$.

Table 5: Results for median of 25 trials (Case C)

\begin{tabular}{|c|c|c|c|c|}
\hline Algorithm & SLL(dB) & $\begin{array}{c}\text { Directivity } \\
(\mathrm{dB})\end{array}$ & $\mathrm{AF}$ at $50^{0}(\mathrm{~dB})$ & $\begin{array}{c}\mathrm{AF} \text { at } 120^{0} \\
(\mathrm{~dB})\end{array}$ \\
\hline DIWO & $\mathbf{- 2 1 . 5 2}$ & $\mathbf{1 1 . 2 8}$ & $\mathbf{- 7 7 . 4 4}$ & $\mathbf{- 7 8 . 9 9}$ \\
\hline IWO & -18.51 & 11.15 & -64.13 & -57.49 \\
\hline PSO & -11.42 & 10.34 & -42.92 & -54.15 \\
\hline DE & -18.05 & 10.96 & -52.53 & -48.47 \\
\hline
\end{tabular}

Table 6: Objective function and $P$-values for Wilcoxon's rank-sum test (Case C)

\begin{tabular}{|c|c|c|c|}
\hline Algorithm & Mean obj. value & Std. Dev & $P$-values \\
\hline DIWO & $\mathbf{0 . 1 7 9 4}$ & $\mathbf{0 . 0 2 2 9}$ & NA \\
\hline IWO & 0.2132 & 0.0496 & $2.538 \mathrm{e}-06$ \\
\hline PSO & 0.3865 & 0.0287 & $7.066 \mathrm{e}-18$ \\
\hline DE & 0.2211 & 0.0345 & $4.059 \mathrm{e}-14$ \\
\hline
\end{tabular}

From Table 5 it is clear that the sidelobe levels are best minimized by our hybrid algorithm in both $50^{\circ}$ and $120^{\circ}$ direction. Moreover Table 6 indicates that the results are statistivally significant also. Combinig these two facts we can say that the required optimal pattern synthesis is best achieved by our proposed DIWO algorithm. The array pattern for Case B and $\mathrm{C}$ are shown in Figure 5 and 6 respectively.

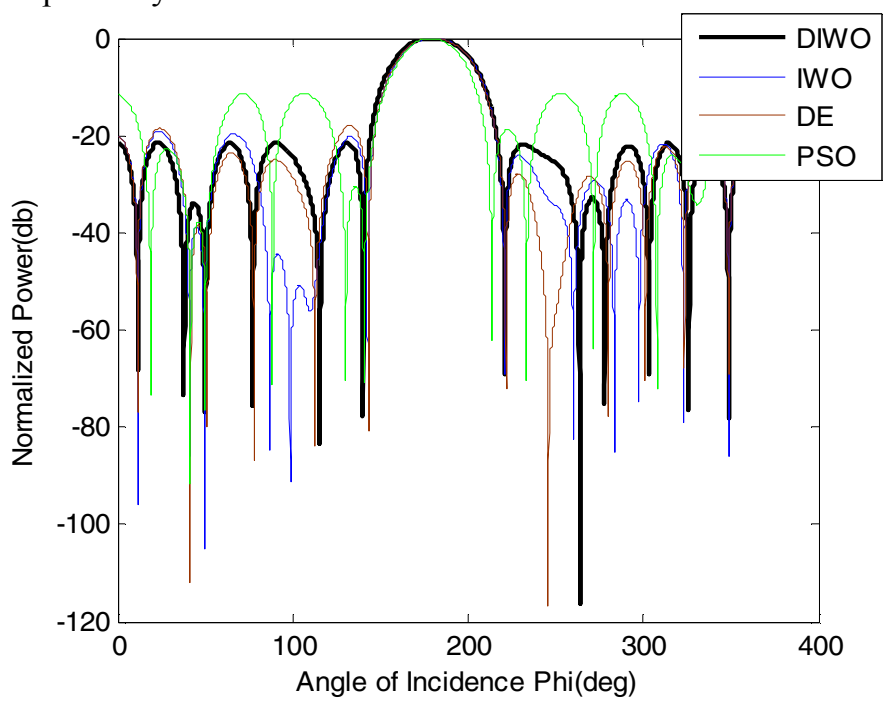

Figure 5: Array Pattern Plot (Case B)

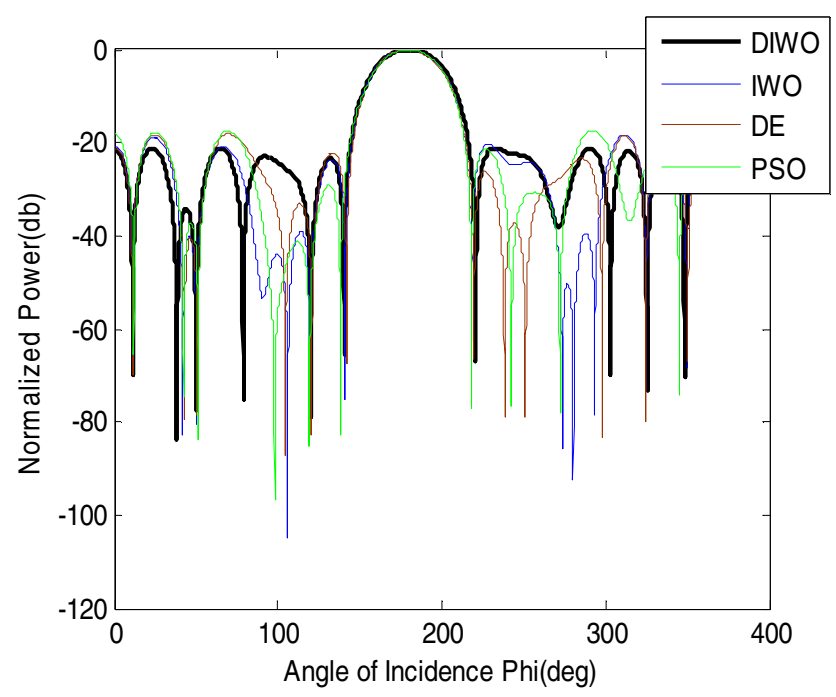

Figure 6: Array Pattern Plot (Case C)

\section{CONCLusions}

Designing circular antenna arrays with minimum SLL, maximum directivity, and also minimum size of the circumference is a challenging optimization problem in electromagnetism. In this article, we proposed a synergistic coupling of DE and IWO and demonstrated 
through simulation experiments, the superiority of the resulting hybrid algorithm over three other state-of-the-art stochastic optimizers in the context of three standard instances of the circular antenna array design problem. We formulated the design problem as an optimization task on the basis of a cost function that takes care of the average side lobe levels, the null control, and the circumference of the array. Our simulation experiments indicated that the hybrid DIWO could comfortably outperform PSO, DE, and GA over 8, 10, and 12 element array design problems based on metrics such as average final accuracy, best obtained design figures of merit (like SLL, directivity, circumference size in terms of wavelength), convergence speed, and robustness, in a statistically significant manner. All these factors together (not just a single factor separately or a combination of some of the factors) have been considered for optimal results in our design problem and this accounts for the significance of this work.

Future research will focus on exploring the design of other array geometries and concentric circular arrays with DIWO and its variants. Also treating the four different components of the cost function given in (10) as a multi-objective optimization problem may prove to be a significant avenue of future investigation, but some problem-specific expert's knowledge may have to be incorporated then for pointing out the best solution from the Pareto-optimal set produced by a multi-objective optimizer.

\section{REFERENCES}

1. L. C. Godara, Ed., Handbook of Antennas in Wireless Communications, CRC, Boca Raton, FL, 2002.

2. S. Chandran (Ed.), Adaptive Antenna Arrays: Trends and Applications, Springer, 2004.

3. G.V. Tsoulos (Ed.), Adaptive Antennas for Wireless Communications, IEEE Press, Piscataway, NJ, 2001

4. Y. Rahmat-Samii and E. Michielssen Eds., Electromagnetic Optimization by Genetic Algorithms. New York: Wiley, 1999.

5. A. Udina, N. M. Martin, and L. C. Jain, "Linear antenna array optimization by genetic means," Third International Conference on Knowledge-Based Intelligent Information Engineering Systems Adelaide, Australia, Sept. 1999.

6. Y. Cengiz and H. Tokat, Linear Antenna Array Design With use of Genetic, Memetic and Tabu Search Optimization Algorithms, Progress In Electromagnetics Research (PIER) C, Vol. 1, 63-72, 2008.

7. W-C. Weng, F. Yang, and A. Z. Elsherbeni, Linear Antenna Array Synthesis Using Taguchi's Method: A Novel Optimization Technique in Electromagnetics, IEEE Transactions on Antennas and Propagation, Vol. 55, No. 3, pp. 723 - 730, March 2007.

8. F. J. Ares-Pena, A. Rodriguez-Gonzalez, E. VillanuevaLopez, and S. R. Rengarajan, "Genetic algorithms in the design and optimization of antenna array patterns," IEEE Transactions on Antennas and Propagation, vol. 47, pp. 506-510, Mar. 1999.

9. Y. B. Tian and J. Qian, "Improve the performance of a linear array by changing the spaces among array elements in terms of genetic algorithm," IEEE Transactions on
Antennas and Propagation., vol. 53, pp. 2226-2230, Jul. 2005.

10. M. M. Khodier and C. G. Christodoulou, "Linear array geometry synthesis with minimum side lobe level and null control using particle swarm optimization," IEEE Transactions on Antennas and Propagation, Vol. 53, No. 8, August 2005.

11. M. I. H. Dessouky, A. Sharshar, and Y. A. Albagory, "Efficient sidelobe reduction technique for small-sized concentric circular arrays," Progress In Electromagnetics Research, PIER 65, 187 - 200, 2006.

12. L. Gurel and O. Ergul, "Design and simulation of circular arrays of trapezoidal-tooth log-periodic antennas via genetic optimization," Progress In Electromagnetics Research, PIER 85, 243 - 260, 2008.

13. M. Dessouky, H. Sharshar, and Y. Albagory, "A novel tapered beamforming window for uniform concentric circular arrays," Journal of Electromagnetic Waves and Applications, Vol. 20, No. 14, 2077 -2089, 2006.

14. M. Panduro, A. L. Mendez, R. Dominguez, and G. Romero, "Design of non-uniform circular antenna arrays for side lobe reduction using the method of genetic algorithms", Int. J. Electron. Commun. (AEU) 60, 713-717, 2006.

15. M. Shihab, Y. Najjar, N. Dib, and M. Khodier, "Design of non -uniform circular antenna arrays using particle swarm optimization", Journal of Electrical Engineering, Vol. 59, No. 4, 216-220, 2008.

16. M. A. Panduro, C. A. Brizuela, L. I. Balderas and D. A. Acosta, "A comparison of genetic algorithms, particle swarm optimization and the differential evolution method for the design of scannable circular antenna arrays", Progress In Electromagnetics Research B, Vol. 13, 171186, 2009.

17. A. R. Mehrabian and C. Lucas, "A novel numerical optimization algorithm inspired from weed colonization," Ecological Informatics, vol. 1, pp. 355-366, 2006.

18. A. R. Mallahzadeh, S. Es'haghi, and A. Alipour, "Design of an E-Shaped Mimo Antenna Using IWO Algorithm for Wireless Application at $5.8 \mathrm{GHz}$ ", Progress In Electromagnetics Research, PIER 90, 187 - 203, 2009.

19. A. R. Mallahzadeh, S. Es'haghi, and H. R. Hassani, Compact U-array MIMO antenna designs using IWO algorithm, International Journal of $R F$ and Microwave Computer-Aided Engineering, Wiley-InterSscience, DOI: 10.1002/mmce.20379, Jul, 2009.

20. S. Karimkashi, and A. A. Kishk, "Invasive weed optimization and its features in electromagnetics," IEEE Transactions on Antennas and Propagation, Vol. 58, Issue 4, pp.1269-1278, April, 2010.

21. A. Basak, S. Pal, S. Das, and A. Abraham, A Modified Invasive Weed Optimization Algorithm for TimeModulated Linear Antenna Array Synthesis, IEEE Congress on Evolutionary Computation (CEC) 2010, WCCI 2010, Barcelona, Spain (Accepted).

22. K. Price, R. Storn, and J. Lampinen, Differential Evolution - A Practical Approach to Global Optimization, Springer, Berlin, 2005.

23. F. Wilcoxon, "Individual comparisons by ranking methods", Biometrics, 1, 80-83, 1945.

24. S. García, D. Molina, M. Lozano, and F. Herrera, "A study on the use of non-parametric tests for analyzing the evolutionary algorithms' behavior: a case study on the CEC'2005 special session on real parameter optimization", Journal of Heuristics, Vol. 15, Issue 6, Pages: 617-644 Dec. 2009. 